\title{
Optimization of laser wavelength, power and pulse duration for eye-safe Raman spectroscopy
}

\author{
Federico Angelini* (D) and Francesco Colao
}

\begin{abstract}
Raising the interest in remote chemical analysis, in particular through Raman and fluorescence spectroscopy, the opportunity of increasing the exposure represents an important step for an easier and more reliable spectrum analysis. However, the European directive 2006/25/EC defines the maximum permitted exposure (MPE) to artificial radiations according to exposure duration, wavelength, coherence of the radiation and beam divergence. Though the Raman cross section scales in general according to the fourth power of the excitation wavelength, promoting the use of deep UV radiation, a synergy between wavelength and exposure time can raise the Raman signal in the near UV or in the near IR if compliance to eye-safety directives is requested. In this work we will analyze the possibilities offered by commercially available components for enhancing the Raman scattering under eye-safe conditions.
\end{abstract}

Keywords: Raman spectroscopy, Standoff identification, Safety directives, Laser exposure, Eye-safe

\section{Introduction}

Laser spectroscopy represents a very useful tool for chemical analysis. In particular, Raman and fluorescence spectroscopy are successfully used in detection of biological in vivo analysis and standoff/proximal detection of unknown substances $[1,2]$. Sometimes the compliance with the regulations about exposure to laser radiations is requested. For example, for counterterrorism or medical diagnostics a key characteristic is the possibility to expose subjects to laser radiation without damaging skin or eyes. Because of the low Raman cross sections, that in many cases cannot be profitably detected and used for identification and classification of materials, a variety of methods have been developed to enhance the signal and allow spectral recognition and identification (Coherent Antistokes Resonant Spectroscopy, Surface Enhanced Raman Spectroscopy, Shifted Excitation Raman Difference Spectroscopy, Resonant Raman Spectroscopy, Waveguide enhaced Raman spectroscopy) [3-7]. Unfortunately, none of these methods work without sample preparation or precise choice of the wavelength, becoming useless for individuation of unknown species and/ or without possibility of handling the target. As a

* Correspondence: federico.angelini@enea.it

ENEA FSN-TECFIS-DIM, via Enrico Fermi, 45, 00044 Frascati, Italy consequence, it is clear that only the single-excitation, non-resonant Raman spectroscopy allows species identification with a minimal exposure of any unknown target, though the return signals are the lowest. The possibility to increase the laser energy used for the analysis, without trespassing the Maximum Permitted Exposure (MPE) is hence a critical point to address in order to improve the backscattered signal and the performances of the subsequent data analyses. The MPE is determined on the basis of the mechanism of damage induced in tissues by the laser radiation: this depends primarily on the wavelength, but the duration of the pulse also impacts on this. Three kinds of effects have been individuated as driving the damaging mechanisms on biological tissues: thermal effects, photochemical effects and non-linear effects. Annex D of the IEC 60825-1 standard [8] describes these mechanisms and discusses the possible damages for eye and skin.

\section{The directive}

The European Directive 2006/25/EC (ED) [9] aims to improve the health and safety of workers exposed to artificial optical radiation by laying down limit values for exposures to eyes and skin. Such directive follows the standards of the International Electrotechnical Commission (IEC) in 
respect of laser radiation and the recommendations of the International Commission on Illumination (CIE) and the European Committee for Standardization (CEN) in respect of non-coherent radiation. With reference to laser radiation it follows the IEC 60825-1 standard, and establishes the laser MPE according to laser wavelength and duration, both in case of continuous and pulsed radiation.

In the present work we will address the problem of finding the best strategy to optimize the Raman signal from a target, still staying within the limits given by the ED, which fixes the MPE to artificial optical radiation, giving detailed prescriptions for the laser emitted light in the range 180 to $3000 \mathrm{~nm}$. Many distinctions are made, according to the type of possible damage on cornea and skin, on the base of exposure duration, wavelength, coherence of the radiation and beam divergence.

In case of a single exposure (i.e. continuous illumination for a time duration T), ED Tables 2.2, 2.3 and 2.4 describe the thresholds and the algorithms for calculating the eye and skin exposures for exposure times from $10^{-13}$ to $10^{4} \mathrm{~s}$, for a laser beam of a given divergence. The averaging area where the energy density must be calculated is also defined, giving the aperture (i.e. diameter) of the circle corresponding to that area.

If the exposure is obtained through multiple pulses, additional conditions must be fulfilled. In this case, the MPE also depends on the number of laser pulses employed within the total time $\mathrm{T}$, so that the possibility to deploy the whole MPE corresponding to the time lapse $\mathrm{T}$ depends on the characteristics of the specific laser in use. This will be better discussed in the following.

In some cases, the MPE depends on the duration of the exposure, while in other it is constant. One can notice that only in the VIS-NIR region $(400-1400 \mathrm{~nm})$ the MPE of skin (SMPE) is different than the MPE of eye (EMPE). Since in most occasions in laser remote sensing it is hard to predict if the laser radiation will hit only skin, in the following the most restrictive rule should be applied, i.e. only the EMPE will be considered. In Fig. 1 the EMPE and SMPE for some selected exposure times are shown for a beam divergence less than $1.5 \mathrm{mrad}$. In the UV region, MPE is independent on the beam divergence $\alpha$.

\section{Single shot}

Figure 1 is adapted after Table 2.2 and shows the single pulse MPE for eye and skin for exposure times ranging from $1 \mathrm{~ns}$ to $10 \mathrm{~s}$, and wavelengths from 180 to $2700 \mathrm{~nm}$. From Fig. 1 it is clear that for Raman spectroscopy the UV region becomes interesting since the MPE can be substantially higher than in the VIS; moreover, the cross section is higher than in the VIS/IR and laser sources,

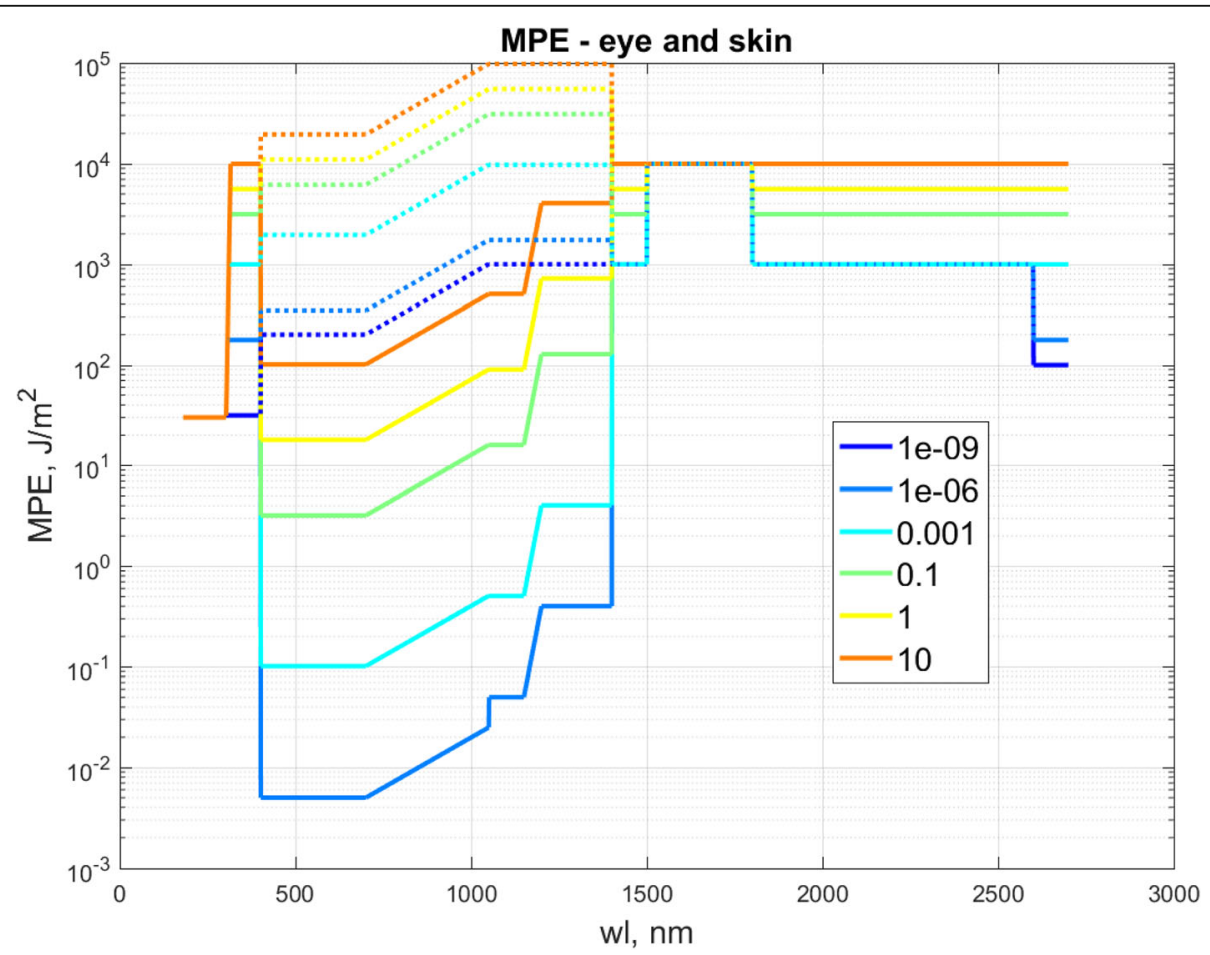

Fig. 1 MPE vs wavelength for some exposure times for $a<1.5 \mathrm{mrad}$. Solid lines refer to eye MPE, while dotted lines refer to skin MPE. Time in legend is expressed in $\mathrm{s}$ 
high efficiency spectrometers, fast and efficient detectors are available.

According to the ED, in the UVC (180-280 nm), UVB $(280-314 \mathrm{~nm})$ and UVA (315-400 nm) regions, substantially different exposures are allowed: below $302 \mathrm{~nm}$ the single-exposure MPE is set at $30 \mathrm{~J} \mathrm{~m}^{-2}$ (i.e. $3 \mathrm{~mJ} \mathrm{~cm}^{-2}$ ), regardless the pulse duration T. Between 302 and 315 $\mathrm{nm}$ there are threshold times (ranging from $2.6 \mathrm{~ns}$ to 1.6 s) under which the MPE (both EMPE and SMPE) grows as a quarter square of the exposure time: $\mathrm{MPE}=5.610^{3}$ $\mathrm{T}^{0.25} \mathrm{~J} \mathrm{~m}^{-2}$; over these times MPE saturates to a fixed value. Over $315 \mathrm{~nm}, \mathrm{EMPE}=5.610^{3} \mathrm{~T}^{0.25} \mathrm{~J} \mathrm{~m}^{-2}$.

Considering UVA radiation, the EMPE values corresponding to exposure times from $1 \mathrm{~ns}$ to $10 \mathrm{~s}$ are listed in Table 1. It is clear that if a single exposure of $1 \mathrm{~s}$ would be possible, the use of UVA region allows an MPE up to 178 times that associated to $1 \mathrm{~ns}$, typical lower limit of the pulse duration in active Q-switched lasers. Below this threshold time, a different regime applies for calculating the MPE, and it lowers as exposure time shortens. For this reason, in this work we can consider $1 \mathrm{~ns}$ as a lower bound for exposure times.

In the regions below 302 and between 1500 and 1800 $\mathrm{nm}$ the MPE is independent with respect of the exposure time, and equals, respectively, 30 and $10^{4} \mathrm{~J} / \mathrm{m}^{2}$.

\section{Multiple shots}

In case of exposure to multiple pulses, a set of additional rules (ED, Table 2.6) must be taken into account:

1. The exposure from any single pulse in a train of pulses shall not exceed the exposure limit value for a single pulse of that pulse duration.

2. The exposure from any group of pulses (or sub-group of pulses in a train) delivered in time $t$ shall not exceed the exposure limit value for time $t$.

3. The exposure from any single pulse within a group of pulses shall not exceed the single-pulse exposure limit value multiplied by a cumulative-thermal correction factor $\mathrm{C}_{\mathrm{p}}=\mathrm{N}^{-0,25}$, where $\mathrm{N}$ is the number of pulses. This rule applies only to exposure limits to protect against thermal injury, where all pulses delivered in less than $\mathrm{T}_{\text {min }}$ are treated as a single pulse.

Thermal injury is only applied at wavelengths longer than $315 \mathrm{~nm}$, where thermal damage is possible; $\mathrm{T}_{\text {min }}$ values are given in Table 2. Although IEC60825-1 standard applies the 3rd requirement only over $400 \mathrm{~nm}$, ED is a little ambiguous since not any clear wavelength range is declared, but only refers to thermal damage. However,

Table 1 Single-pulse MPEs for several exposure times in the UVA region (315-400 $\mathrm{nm}$ )

\begin{tabular}{lcccccccc}
\hline Time & $1 \mathrm{~ns}$ & $1 \mathrm{us}$ & $100 \mathrm{us}$ & $1 \mathrm{~ms}$ & $0.01 \mathrm{~s}$ & $0.1 \mathrm{~s}$ & $1 \mathrm{~s}$ & $10 \mathrm{~s}$ \\
\hline MPE $\left(\mathrm{J} / \mathrm{m}^{2}\right)$ & 31.5 & 177 & 560 & 995 & 1771 & 3149 & 5600 & 9958 \\
\hline
\end{tabular}

Table $2 T_{\min }$ as a function of wavelength. Pulses closer than $T_{\min }$ should be considered as a single pulse

\begin{tabular}{lll}
\hline$\lambda_{\min }$ & $\begin{array}{l}\lambda_{\max } \\
\mathrm{nm}\end{array}$ & $\begin{array}{l}\mathrm{T}_{\min } \\
\mathrm{sm}\end{array}$ \\
\hline 315 & 400 & $10^{-9}$ \\
400 & 1050 & $18 \cdot 10^{-6}$ \\
1050 & 1400 & $50 \cdot 10^{-6}$ \\
1400 & 1500 & $10^{-3}$ \\
1500 & 1800 & 10 \\
1800 & 2600 & $10^{-3}$ \\
2600 & $10^{6}$ & $10^{-7}$ \\
\hline
\end{tabular}

since Table A.1 of IEC60825-1 and Table ED 2.1 declare the occurrence of thermal damage for eye also in the $180-400 \mathrm{~nm}$ region, the most conservative calculation must also consider the requirement 3.

It is important to notice that, in the UVA region, any laser available today will provide pulses to be considered separately, while in the region $1500-1800 \mathrm{~nm} \mathrm{~T}_{\min }$ is so long that any train of laser pulses can be considered as a single pulse, leading to a greater MPE since the correction factor $C_{p}$ equals 1 .

While rules 1 and 2 are easy to merge between them, rule 3 complicates the scenario since the maximum energy density per pulse depends on the number of pulses. Since each laser can deliver a maximum energy per pulse (MEP) in a range of Pulse Repetition Rates (PRR), a best combination of pulses and energy will exists for each specific device. In the next section a comparison of the performances among a selection of different commercial laser models will be presented.

\section{Enhancing the Raman signal}

Starting from the MPE values discussed so far, we will calculate now the Raman signals associated to different wavelengths, exposure times and laser types. We must consider that, in general, the Raman cross section of a molecule scales with the fourth power of the wavelength. This is true in regions free from resonances, then in general in the near UV, VIS and IR region. However, since the energy of each photon is proportional to its frequency, the net effect of using a pulse of a given energy on Raman signal is a dependence on the third power of the wavelength. Figure 2 shows a theoretical Raman signal (relative to the one at $180 \mathrm{~nm}$ ) coming from a target where the total MPE has been deposited by a single pulse.

Two regions appear particularly appealing: the UVA-B and the IR between 1500 and $1800 \mathrm{~nm}$. In the first one, many commercial laser lines are available (see Table 3) both fixed and tunable. In this region the best performance could be achieved using a continuous laser $(\mathrm{CW})$ to accumulate signal for up to $10 \mathrm{~s}$ (when MPE saturates 


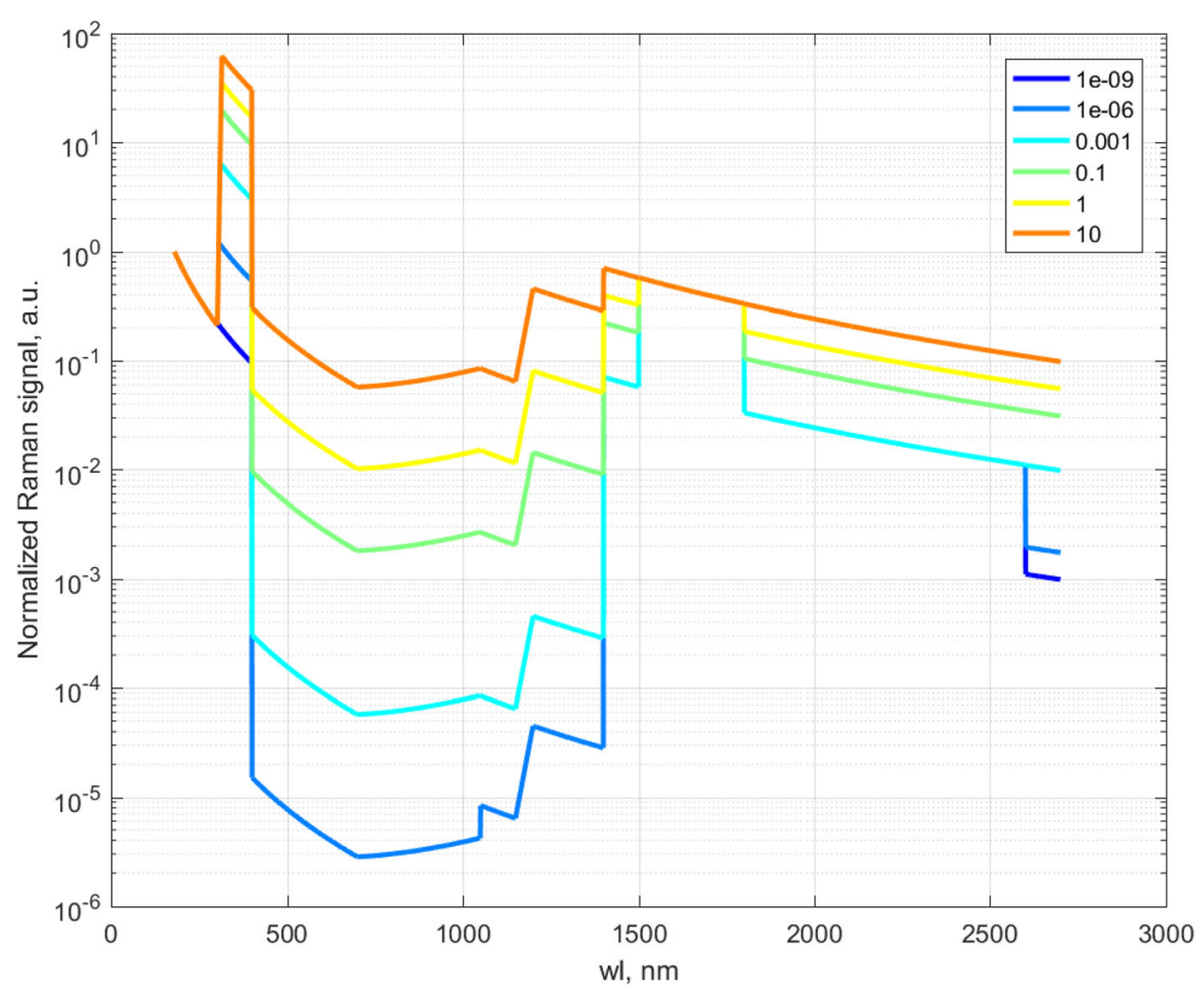

Fig. 2 The expected Raman signal, normalized to 1 at $180 \mathrm{~nm}$, for exposure times between $1 \mathrm{~ns}$ and $10 \mathrm{~s}$

at $10000 \mathrm{~J} / \mathrm{m}^{2}$ ). With pulsed laser, a different strategy must be adopted, and it will described in the next section since it makes use of multiple exposure.

In the UV region, fluorescence may represent a very limiting factor for UV-VIS Raman investigation. In fact, the fluorescence signal can be often so intense to mask the Raman signal: although the fluorescence bands are usually much broader than Raman lines and in theory could be filtered out by a digital filter, the shot noise associated to this intense signal can cause fluctuations of the same order of magnitude of the most intense Raman lines. A possibility of rejecting fluorescence in the UV-VIS region is based on time-resolved spectroscopy, because fluorescence usually happens on time scales

Table 3 Some available laser lines in the UVB-UVA region

\begin{tabular}{llll}
\hline Active material & Laser type & Region & $\mathrm{Nm}$ \\
\hline $\mathrm{XeCl}$ & Excimer & UVB & 308 \\
$\mathrm{Ne}+$ & lon & UVA & 332 \\
$\mathrm{XeF}$ & Excimer & UVA & 351 \\
$\mathrm{Nd}: Y A G$ tripled & Solid state & UVA & 355 \\
Ti:Sa tripled & Solid state & UVB-UVA & $235-330$ \\
Ti:Sa doubled & Solid state & UVB-UVA & $240-330$ \\
Alexandrite doubled & Solid state & UVA & $350-400$ \\
Dye & dye & UVA-UVB & 200-400 \\
\hline
\end{tabular}

longer than Raman effect [10, 11]. Using short laser pulses $(<1 \mathrm{~ns})$ and fast gated ICCD $(\sim 2 \mathrm{~ns})$ it might be possible to cut off most of the fluorescence signal. However, the MPE corresponding to subnanosecond pulses is very low, since from ED Table 2.2 the power of pulses shorter than $1 \mathrm{~ns}$ is set to $10^{10} \mathrm{~W} / \mathrm{m}^{2}$ (from 180 to 400 $\mathrm{nm}$ ). This forces the MPE to scale linearly in this region, leading to very weak signals.

In spite of the reduced Raman cross section, also the region 1500-1800 nm seems appealing, at least from a theoretical point of view. In this region, in fact, the MPE is very high, and then a large amount of photons can be delivered on the target: moreover, as already observed, the MPE does not depend on exposure time. This means that, in case of single exposure, if short pulses must be employed (i.e. fast dynamics of a process) the Raman signal in this region can also overcome those from UVB for pulse lenghts shorter than about $1 \mu \mathrm{s}$, as visible in Fig. 2.

Another advantage of the IR lies in the absence of fluorescence signal. In fact, fluorescence happen at longer wavelengths than the excitation one.

The main drawback of IR Raman spectroscopy comes from the efficiency of optics and detectors, whose quantum efficiencies are not comparable to those operating in the UV region. In addition, the high absorption of IR radiation by water may also limit the utility of IR 
Raman spectroscopy for biological samples, reducing the scattering volume.

Depending on the area under investigation, cost, dimensions and weight admissible for the instrument, it is possible that no $\mathrm{CW}$ lasers can reach the power to deploy the entire $\mathrm{MPE}_{\mathrm{T}}=\operatorname{MPE}(\mathrm{T})$. In this case it is possible to fall back on pulsed lasers. With pulsed lasers, many short pulses can be sent within a longer time lapse. In any case, using different pulse lenghts means that the CCD exposure should lengthen as well, leading to different noise levels: the different signals should be compared to the corresponding noise levels $[12,13]$.

Each light measurement leads to an intrinsic error due to the discrete nature of photons: the intensity fluctuations are described by the Poisson statistics and hence the variance associated to a signal (both spectral line, dark or background) equals the expected value. In this case the ratio $S$ / $N=S / \sqrt{S}=\sqrt{S}$ grows together with $\mathrm{S}$ (i.e. the brighter the signal, the lowest the relative error).

Since the overall value of the noise depends actually on a variety of factors such as temperature, dark noise, readout noise and background illumination, a thorough discussion on Raman signal-to-noise ratio is beyond the aim of this work; however, as a rule of thumb we can say that if the detector noise does not grow dramatically with integration time the best strategy could be represented by sending more pulses in times as long as $10 \mathrm{~s}$. For example, in cooled, state-of-the-art, CCD cameras the main fluctuation comes from readout noise rather than dark noise: in fact, noise is almost constant in exposure up to several seconds. From a practical point of view, it is useful to define the noise as the standard deviation of the intensity in a region where no spectral lines are present, the SNR is here computed as the ratio between the peak signal by the noise as above defined.

The performances achievable by the use of repetitively pulsed lasers within a time lapse $\mathrm{T}$ will be now analyzed.

First of all, given the wavelength, $\mathrm{T}_{\text {min }}$ must be checked to establish whether the pulses can be considered as a single pulse or not. If not, with such pulsed laser, the single-shot MPE changes as described before and the most restrictive of the three requirements listed before must be fulfilled. The third one involves the number of pulses sent in the total measurement time: if $\mathrm{N}$ pulses of length $\mathrm{t}$ are sent within an interval $\mathrm{T}$, the single-shot MPE $\left(\mathrm{MPE}_{\mathrm{t}}\right)$ shall be decreased by the factor $\mathrm{Cp}=\mathrm{N}^{-0.25}$. It is worth notice that the MPE for single pulse is specific for each laser since the pulse length is imposed by the laser constructive features; on the other hand, at least one between single-shot energy and PRR can be changed to match the energy needed to fill the $\mathrm{MPE}_{\mathrm{T}}$ with pulses not exceeding $\mathrm{MPE}_{\mathrm{t}} \cdot \mathrm{C}_{\mathrm{p}}$ :

$$
\begin{aligned}
& M P E_{T}=N \cdot M P E_{t} \cdot C p=N \cdot M P E_{t} \cdot N^{-1 / 4}=M P E_{t} \cdot N^{3 / 4} . \\
& \text { Hence, } N=\left(\frac{M P E_{T}}{M P E_{t}}\right)^{4 / 3} .
\end{aligned}
$$

In the special case where $\operatorname{MPE}(\mathrm{t})=5600 \mathrm{t}^{1 / 4} \mathrm{~J} / \mathrm{m}^{2}$ (i.e. in the UVA region and $1 \mathrm{~ns}<(\mathrm{T}, \mathrm{t})<10 \mathrm{~s})$, the term $\mathrm{MPE}_{\mathrm{T}} / \mathrm{MPE}_{\mathrm{t}}$ simplify and $N=\left(\frac{T}{t}\right)^{1 / 3}$.

As the MPE is an energy density, care has to be taken to rescale the laser energy density to the spot area, because if a large spot is used, the energy per pulse might be too low to fill the entire amount of MPE. In this case, a larger number of pulses must be employed. Let $J_{0}$ be the maximum energy density available at each pulse. The number of pulses required to fill the $M P E_{T}$ will be $N=\frac{M P E_{T}}{J_{0}}$, i.e.: $P R R=\frac{M P E_{T}}{J_{0} \cdot T}$, as far as the condition $\mathrm{J}_{0}<$ $\mathrm{MPE}_{\mathrm{t}} \mathrm{C}_{\mathrm{p}}$ is satisfied. In the special case where MPE $=$ $5600 \mathrm{t}^{1 / 4} \mathrm{~J} / \mathrm{m}^{2}$, again these expressions become simpler: PRR $=\frac{5600}{J_{0}} T^{-3 / 4} \mathrm{~Hz}$.

From what discussed so far, it is evident that a laser with an average power of $\mathrm{MPE}_{\mathrm{T}} / \mathrm{T}$ is requested to deploy all the $\mathrm{MPE}_{\mathrm{T}}$ (for each square centimeter of the laser spot). Moreover, a laser with high PRR and low peak power is preferable to avoid the risk of saturating the single shot $\mathrm{MPE}_{\mathrm{t}}$ : in fact, a laser deploying its power through few powerful pulses is likely unable to fill the $\mathrm{MPE}_{\mathrm{T}}$.

Referring to the region $315-400 \mathrm{~nm}$ and $1 \mathrm{~ns}<\mathrm{T}<$ $10 \mathrm{~s}$, Fig. 3 shows the fraction of the total MPE available for $\mathrm{T}=0.01,0.1,1$ and $10 \mathrm{~s}$, using pulses $1 \mathrm{~ns}$ long, as a function of the PRR and $\mathrm{J}_{0}$. Dotted lines represents loci where $\mathrm{PRR}^{*} \mathrm{~J}_{0}=$ constant, i.e. curves of constant power. In general, the power $\mathrm{W}$ required to fill the $M P E_{T}$ is given by $\mathrm{MPE}_{\mathrm{T}} / \mathrm{T}$ for each square centimeter of the beam area. In this spectral region $\mathrm{W} \geq 560 \mathrm{~T}^{-3 / 4} \mathrm{~mW} / \mathrm{cm}^{2}$. On the other hand, the inverse of this formula may give the minimum time required to obtain the complete filling of the MPET given the laser power: $\mathrm{T}=(0.560 / \mathrm{W})^{4 / 3}$ (where $\mathrm{W}$ is in $\mathrm{W}$ and $\mathrm{T}$ in $\mathrm{s}$ ). It might be useful to remember form Table 1 that $M P E_{T}$ for $T=1 \mathrm{~s}$ equals $560 \mathrm{~mJ} /$ $\mathrm{cm}^{2}$ while for $\mathrm{T}=0.1 \mathrm{~s}$ equals $315 \mathrm{~mJ} / \mathrm{cm}_{2}$. The plots refer to $1 \mathrm{~ns}$ pulses since this time entails the most restrictive $M P E_{t}$ : longer laser pulses will automatically fulfill the single-pulse requirements.

In the $\mathrm{J}_{0}-\mathrm{PRR}$ space, the locus of points where $\mathrm{J}_{0}=$ $\mathrm{MPE}_{\mathrm{t}} \cdot \mathrm{C}_{\mathrm{p}}$ (plotted as a black line in Fig. 3) splits the plane in two regions: this locus can be called the discriminant line. Above this line, $\mathrm{J}_{0}$ is considered to be trimmed to $\mathrm{MPE}_{\mathrm{t}}$, and raising the PRR only increases linearly the exposure. Below, the whole $\mathrm{J}_{0}$ is available, and raising the PRR increments the exposure as isopower lines are crossed, until the discriminant or the $100 \%$ line is reached. In this space each laser can be represented as a point of coordinates corresponding to the max PRR (PRR ${ }_{\text {MAX }}$ ) and max Energy per pulse $E_{\text {MAX }}$ ( $\mathrm{J}_{\text {MAX }}$ if normalized to the beam area): $\mathrm{E}_{\text {MAX }} \cdot \mathrm{PRR}_{\mathrm{MAX}}$ will also determine the max laser power. 


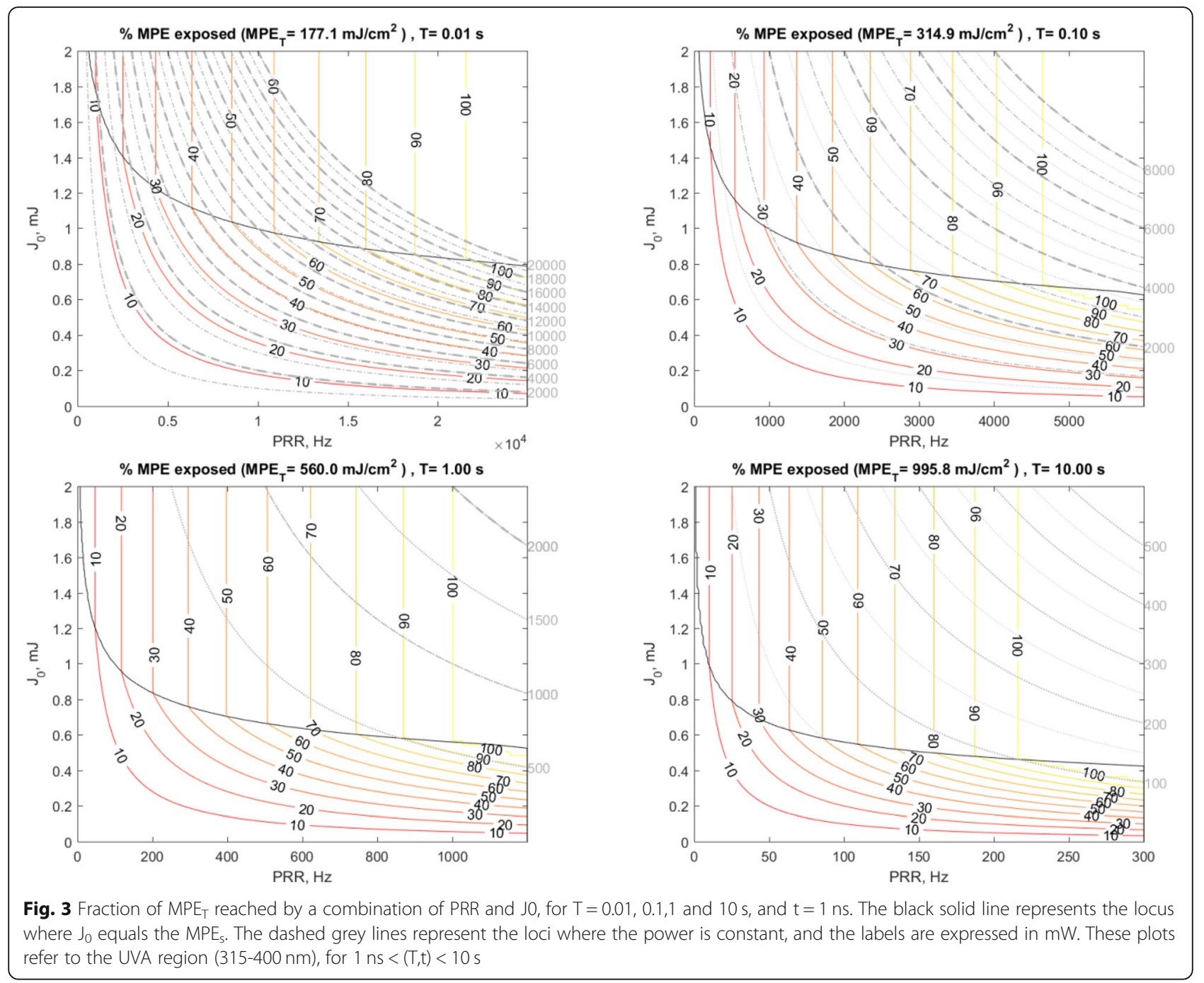

Table 4 Fraction of $\mathrm{MPE}_{\mathrm{T}}$ and expected Raman signal reached by some types of lasers

\begin{tabular}{|c|c|c|c|c|c|c|c|c|c|c|c|c|c|}
\hline \multicolumn{5}{|c|}{ Laser characteristics } & \multicolumn{4}{|c|}{ Fraction of $\mathrm{MPE}_{\mathrm{T}}(\%)$} & \multicolumn{4}{|c|}{ Expected Raman signal (a.u.) } & \multirow[t]{2}{*}{ Laser type } \\
\hline$\overline{w l}$ & power & PRR & $E_{0}$ & $t$ & $\mathrm{~T}(\mathrm{~s})$ & & & & $\mathrm{T}(\mathrm{s})$ & & & & \\
\hline $\mathrm{nm}$ & W & $\mathrm{Hz}$ & $\mathrm{mJ}$ & ns & 0.01 & 0.1 & 1 & 10 & 0.01 & 0.1 & 1 & 10 & \\
\hline 355 & 0.2 & 50 & 4 & 11 & 1 & 6 & 19 & 61 & 0.28 & 2.69 & 15.1 & 85.0 & Lamp pumped, tripled Nd:YAG \\
\hline 355 & 0.7 & 100 & 7 & 12 & 3 & 10 & 33 & 100 & 0.82 & 4.62 & 26.0 & 140 & Diode pumped, tripled Nd:YAG \\
\hline 355 & 4 & $410^{4}$ & 0.1 & 2 & 23 & 100 & 100 & 100 & 5.61 & 44.2 & 78.5 & 140 & Diode pumped, tripled Nd:YAG \\
\hline 355 & 8 & $510^{4}$ & 0.16 & 25 & 45 & 100 & 100 & 100 & 11.2 & 44.2 & 78.5 & 140 & Diode pumped, tripled Nd:YAG \\
\hline 351 & 15 & 750 & 20 & 18 & 17 & 52 & 100 & 100 & 4.26 & 24.0 & 81.2 & 144 & Excimer, $\mathrm{XeFI}$ \\
\hline 266 & 0.08 & 20 & 4 & 11 & 100 & 100 & 100 & 100 & 1.00 & 1.00 & 1.00 & 1.00 & Lamp pumped, quadrupled Nd:YAG \\
\hline 405 & 0.5 & CW & 500 & $10^{9}$ & 100 & 100 & 100 & 100 & 0.01 & 0.03 & 0.17 & 0.96 & CW diode laser \\
\hline 325 & 0.1 & $C W$ & 100 & $10^{9}$ & 1 & 3 & 18 & 100 & 0.18 & 1.83 & 18.3 & 182 & He-Cd CW laser \\
\hline 1570 & 1.5 & 30 & 50 & 6 & 2 & 15 & 100 & 100 & 0.02 & 0.24 & 1.62 & 1.62 & OPO-shifted Er:glass \\
\hline
\end{tabular}


To compare different lasers, we performed calculations of the theoretical Raman return from a sample target for a variety of laser categories, whose typical characteristics have been chosen according to the active medium and the pumping technique. Table 4 shows the fraction of effective by admitted $\mathrm{MPE}_{\mathrm{T}}$ and the expected maximum signals obtained by each laser category, for several exposure time ranging between $0.01 \mathrm{~s}$ to $10 \mathrm{~s}$ normalized to the signal of a $266 \mathrm{~nm}$ device reaching the corresponding MPE. All the calculations have been performed for a spot area of 1 $\mathrm{cm}^{2}$ and for a collimated beam. If different beam size is employed, the energy density will change accordingly. If $\mathrm{J}_{0}>\mathrm{MPE}_{\mathrm{t}}$ the laser should be attenuated, or the beam expanded in order not to trespass the single shot MPE; otherwise the beam could be narrowed to match the $\mathrm{MPE}_{\mathrm{t}}$. In any case one must take care of the homogeneity of the beam, since ED sets the limiting aperture as the circular area over which irradiance and radiant exposure are averaged. In the UV region, such aperture equals $1 \mathrm{~mm}$ for pulses shorter than $0.3 \mathrm{~s}$ [9, Table 2.2]. If the beam is not collimated, the divergence of the beam affects the calculation of MPE in the spectral range $400-1400 \mathrm{~nm}$.

From Table 4 it is evident that also in case where the total laser power is given, devices with high repetition rate and low peak power can fill the MPE much easier than lasers delivering high energy per pulse, at least for a beam area of $1 \mathrm{~cm}^{2}$. If the area increases, more intense pulses can still fill the MPE, changing the results. On the other hand, smaller areas allow less powerful lasers to reach high fraction of the MPE. In general, however, shorter acquisition times require more powerful lasers. As a consequence, if the dynamics of the process is not very fast, raising the integration time as much as possible should be preferred.

Long exposures ( $\mathrm{T}>1 \mathrm{~ms}$ ) can be exploited also in the IR $(\lambda>1400 \mathrm{~nm})$ region, where the MPE is the same as in the UVB region, and between 1500 and 1800 reaches $1000 \mathrm{~mJ} / \mathrm{cm}^{2}$ regardless the pulse length. Nevertheless, the Raman cross section, for a given energy, is here much lower than in the UVB, (about 80 times between 355 and $1540 \mathrm{~nm}$ ), and the water absorption may limit the advantage of high MPE, mainly for thick samples with high water content. Moreover, currently the narrow assortment of high power laser devices (to the autors' knowledge, only OPO-shifted Er:glass at 1570 or diode fiber-amplified at $1550 \mathrm{~nm}$ are available in this region), efficient optics and detectors strongly limits the practical implementation of this solution, nevertheless leaving open this possibility for the next generation of laser and detectors.

\section{Conclusions}

The UVA region seems the most suitable region to obtain high Raman signal with eye-safe laser radiation, both with continuous and pulsed laser, provided that pulse energy and repetition rate are in agreement with the constraint for repetitive exposure.

Using repetitive exposures in time windows up to $10 \mathrm{~s}$, the MPE can grow with respect to a single pulse depending on the spectral region considered. Of course, if time is increased the detector acquisition time must be enhanced as well. With cooled CCD devices and at low and very low signal level, the background illumination must be kept as low as possible in order to have a negligible contribution, then to limit readout noise then the best strategy might consist in accumulating all the pulses in one single frame.

Calculations were made considering $1 \mathrm{~cm}^{2}$ laser spot. If the target has a different area, the laser power should be scaled accordingly. Larger spots imply stronger signals, but it would be hard to find lasers powerful enough to fill the whole MPE, especially for short integration times. However, this is a purely technological limit: as more powerful lasers become available on the market, it will be easier to fill larger areas with higher energies.

As last remark it is worth notice that whenever the substrate fluorescence is an issue, the $1500-1800 \mathrm{~nm}$ range may represent an interesting alternative, although the unavailability of efficient and low noise detectors and optics, as well as laser sources can limit the final performances.

\section{Abbreviations}

CCD: Charge Coupled Device; ED: European Directive 2006/EC/25; MPE: Maximum Permitted Exposure; PRR: Pulse Repetition Rate

\section{Acknowledgements}

The Authors wish to acknowledge the colleagues Dr. Luca Fiorani and Dr. Valeria Spizzichino for their valuable comments and suggestions.

\section{Funding \\ The work was realized within the project EXTRAS, funded by NATO Science for Peace and Security (SPS) programme.}

Availability of data and materials

All the data supporting the conclusions of this article come from the 2006/ $\mathrm{EC} / 25$ directive. The data associated to the listed laser categories can be found on the websites of many laser manufacturers.

\section{Authors' contributions \\ FA performed the numerical calculations; FC developed the formal calculations. Both authors analyzed the data and wrote the manuscript. Both authors read and approved the final manuscript.}

Competing interests

The authors declare that they have no competing interests.

\section{Publisher's Note}

Springer Nature remains neutral with regard to jurisdictional claims in published maps and institutional affiliations. 
Received: 13 December 2018 Accepted: 11 March 2019

Published online: 21 March 2019

\section{References}

1. Hanlon, E.B., Manoharan, R., Koo, T., Shafer, K.E., Motz, J.T., Fitzmaurice, M., Kramer, J.R., Itzkan, I., Dasari, R.R., Feld, M.S.: Prospects for in vivo Raman spectroscopy. Phys. Med. Biol. 45(2), R1 (2000)

2. Almaviva, S., Angelini, F., Chirico, R., Palucci, A., Nuvoli, M., Schnuerer, F., Schweikert, W., Romolo, F.S.: Eye-safe UV Raman spectroscopy for remote detection of explosives and their precursors in fingerprint concentration. In: Optics and Photonics for Counterterrorism, Crime Fighting, and Defence X; and Optical Materials and Biomaterials in Security and Defence Systems Technology XI, vol. 9253, p. 925303. International Society for Optics and Photonics (2014)

3. Maker, P.D., Terhune, R.W.: Study of optical effects due to an induced polarization third order in the electric field strength. Phys. Rev. 137(3A), A801 (1965)

4. Fleischmann, M., Hendra, P.J., McQuillan, A.J.: Raman spectra of pyridine adsorbed at a silver electrode. Chem. Phys Lett. 26(2), 163-166 (1974)

5. Shreve, A.P., Cherepy, N.J., Mathies, R.A.: Effective rejection of fluorescence interference in Raman spectroscopy using a shifted excitation difference technique. Appl. Spectrosc. 46(4), 707-711 (1992)

6. Albrecht, A.C.: On the theory of Raman intensities. J. Chem. Phys. 34(5), 1476-1484 (1961)

7. Raza, A., Clemmen, S., Wuytens, P.C., Muneeb, M., Daele, M.V., Dendooven, J., Detavernier, C., Skirtach, A., Baets, R.: ALD assisted nanoplasmonic slot waveguide for on-chip enhanced Raman spectroscopy. Appl. Phys. Lett. 3(11), 116105

8. IEC 60825-1 Ed. 2.0 (2007); Safety of laser products - Part 1: Equipment classification and requirements

9. https://eur-lex.europa.eu/legal-content/EN/TXT/?uri=CELEX\%3A02006L002520140101

10. Beechem, J.M., Brand, L.: Time-resolved fluorescence of proteins. Annu. Rev. Biochem. 54(1), 43-71 (1985)

11. Suhling, K., French, P.M., Phillips, D.: Time-resolved fluorescence microscopy. Photochem. Photobiol. Sci. 4(1), 13-22 (2005)

12. O'Malley, M.J., O'Mongain, E.: Charge-coupled devices: frame adding as an alternative to long integration times and cooling. Opt. Eng. 31(3), 522-527 (1992)

13. Dussault, D. and Hoess, P., 2004. Noise performance comparison of ICCD with CCD and EMCCD cameras. In Infrared Systems and Photoelectronic Technology (Vol. 5563, pp. 195-205). International Society for Optics and Photonics

\section{Submit your manuscript to a SpringerOpen ${ }^{\circ}$ journal and benefit from:}

- Convenient online submission

- Rigorous peer review

- Open access: articles freely available online

- High visibility within the field

- Retaining the copyright to your article

Submit your next manuscript at $\boldsymbol{\nabla}$ springeropen.com 